\title{
Quantum Computation and Quantum
}

Information by Micheal A. Nielsen and Isaac L. Chuang. (2002) Cambridge University Press

One of the most promising scientific developments of last century was the Computers. Computers of initial days were of very large size consisting of vacuum tubes and valves. This has taken over by semiconductor and transistors which were of smaller size and more efficient. The rapid growth in the semiconductor industry has led to the present form computer on our desktop. This has initiated the questions about the ultimate limit of this development. As the size of computer chip is decreasing, it has been predicted by Moor's law that within next twenty year, the size of a single bit will be of the order of a single atom. Physical laws governing the atomic phenomena, such as quantum mechanics, are very different from macroscopic laws. So the computers operating on atomic level will not be same as present days computers. This possibility has opened a completely new field of Quantum Computation. This book is a comprehensive introduction to the main ideas and techniques of the field of quantum computation and quantum information. Until now, the rapid rate of progress and its cross-disciplinary nature have made it difficult for newcomers to the field to obtain a broad overview of the most important techniques and results.

Michael Nielsen and Isaac Chuang ask the question: what are the ultimate physical limits to computation and communication? They describe in detail such remarkable effects as fast quantum algorithms, quantum teleportation, quantum cryptography, and quantum error-correction. A wealth of accompanying figures and exercises illustrate and develop the material in more depth. A special feature is the futorial introductions to quantum mechanics and computer science included in Part I of the book, ensuring that the subject is accessible even without a background in physics or computer science. In Part II, the authors present quantum computation, and describe what a quantum computer is, how it can be used to solve problems 
faster than familiar 'classical' computers, and the real-world implementation of quantum computers. Part III concludes the book with an in-depth treatment of quantum information, explaining how quantum states can be used to perform remarkable feats of communication, together with a discussion of how it is possible to protect quantum states against the effects of noise.

This exciting book will be of interest to beginning graduate students and researchers in physics, computer science, mathematics, and electrical engineering with an interest in quantum computation and quantum information, and may be used as a text in courses on the subject.

\author{
Neerai Sinha \\ Department of Physics \\ IISC, Bangalore-560012 \\ INDIA
}

